\title{
In memoriam Jean-Pierre Lautridou (1938-2010)
}

Jean-Pierre Coutard et Dominique Cliquet

\section{OpenEdition}

\section{Journals}

Édition électronique

URL : http://journals.openedition.org/rao/1393

DOI : $10.4000 /$ rao.1393

ISSN : 1775-3732

\section{Éditeur}

Presses universitaires de Rennes

\section{Édition imprimée}

Date de publication : 31 décembre 2010

Pagination : 211-215

ISBN : 978-2-7535-1383-9

ISSN : 0767-709X

\section{Référence électronique}

Jean-Pierre Coutard et Dominique Cliquet, «In memoriam Jean-Pierre Lautridou (1938-2010) », Revue archéologique de l'Ouest [En ligne], 27 | 2010, mis en ligne le 25 février 2012, consulté le 05 décembre 2020. URL : http://journals.openedition.org/rao/1393 ; DOI : https://doi.org/10.4000/rao.1393

Ce document a été généré automatiquement le 5 décembre 2020

Tous droits réservés 


\title{
In memoriam Jean-Pierre Lautridou (1938-2010)
}

\author{
Jean-Pierre Coutard et Dominique Cliquet
}

1 Il est toujours émouvant et délicat d'écrire sur une personne qui vient de nous quitter et cela devient encore plus difficile lorsqu'il s'agit d'un collègue proche et ami avec lequel on a travaillé pendant des dizaines d'années sur des programmes de recherche communs. Il ne faut pas la trahir. Avant de cerner la carrière scientifique de Jean-Pierre Lautridou, essayons de décrire l'homme qu'il était. Qu'est qu'un bon chercheur? C'est sans nul doute quelqu'un qui sait recueillir soigneusement des données, émettre des hypothèses, les vérifier soit sur le terrain, soit par des expériences en laboratoire et en tirer des enseignements mais c'est aussi connaître et apprécier le travail de ses prédécesseurs, savoir dialoguer avec autrui, avoir l'art de la communication. Nous pouvons affirmer que Jean-Pierre Lautridou c'était tout cela. D'une grande gentillesse, toujours disponible, souvent généreux vis-à-vis de ses collègues et de ses étudiants, faisant part sans réserve de ses acquis scientifiques, il n'est pas étonnant que, dès le début de sa carrière, il a tissé très vite, autour de lui, un large réseau de relations à la fois scientifiques et amicales, tant en France qu'à l'étranger. De même, il a toujours trouvé des gens heureux de travailler avec lui pour l'organisation des nombreux colloques et des non moins nombreuses excursions scientifiques qu'il prit en charge. C'est également cet état d'esprit qui lui permit la création de groupes de travail informels et efficaces, Groupe Seine, puis, avec Ph. Gibbard, Groupe Manche. Il était un excellent animateur d'équipes. Il aimait les travaux collectifs menés dans une atmosphère de franche camaraderie. Aussi, face aux rares situations conflictuelles qu'il eut à gérer, l'avons-nous senti fortement peiné et quelque peu désemparé. Quant au respect des anciens auteurs, que de nos jours, certains tendent à oublier, il était inné chez lui. Il suffit de voir comment, pour la moindre coupe du Pays de Caux, il a recherché non seulement dans les grandes revues mais aussi dans les publications des nombreuses sociétés savantes, si actives $\mathrm{au} \mathrm{XIX}^{\mathrm{e}}$ siècle et dans la première moitié du $\mathrm{xx}^{\mathrm{e}}$ siècle, qui, universitaire ou érudit local, avait écrit les premières lignes de description, fourni le premier croquis, tout cela pour donner à chacun sa juste place dans le long parcours d'acquisition des connaissances. Certains ont été parfois surpris 
du nombre élevé d'auteurs cités pour des articles de synthèse. Là encore la parfaite honnêteté scientifique de Jean-Pierre le conduisait à citer une personne même si sa contribution était très modeste. Nous en avons fait l'expérience.

2 Tout ceux qui l'ont connu se souviendront de ses exposés d'une clarté exemplaire tant sur le terrain que lors de colloques. Certains très brillants témoignaient de sa passion pour ses domaines d'étude. Jean-Pierre savait faire, aussi, d'excellents exposés en anglais, langue qu'il maitrisait particulièrement bien. Il n'est donc pas surprenant qu'il ait été sollicité pendant de nombreuses années à donner des enseignements dans le DEA de Préhistoire de Paris dirigé par H. de Lumley, le DEA de Géographie de l'Université de Caen et enfin le DESS Environnement, Sols, Eaux de Caen - Rouen et à organiser un certain nombre de séminaire spécialisés. Après sa nomination comme Directeur de recherche en 1984, il dirigera ou assurera la cotutelle de plus d'une quinzaine de thèses auxquelles s'ajoutent deux habilitations à diriger des recherches. Il accueillera un grand nombre de stagiaires à Caen dans son laboratoire de cryoclastie et aidera beaucoup de collègues d'universités françaises et étrangères à réaliser des expériences dont les résultats ont été publiés dans leurs thèses respectives.

3 Beaucoup d'associations et de commissions scientifiques étaient fières de le compter au nombre de leurs membres, en particulier l'Association française pour l'Etude du Quaternaire pour laquelle il a beaucoup œuvré et dont il fut le président de 1990 à 1994.

Jean-Pierre Lautridou, est connu comme étant l'homme de Saint-Romain-de-Colbosc, chef-lieu de canton situé dans le Pays de Caux, en Seine-Maritime. Cela n'est du, ni la faute à Voltaire ni à celle de Rousseau contrairement à ce qui est dit dans un air célèbre mais à la présence, dans cette commune, à l'époque, d'une fort belle coupe de loss qu'il nous invitera, à plusieurs reprises, à venir admirer, si possibles munis de pelles, de pioches et de raclettes. Ce site fait l'objet d'une mise en valeur, gageons que les panneaux inhérents à l'histoire du gisement quaternaire et témoignant d'occupations paléolithiques, citent les travaux de Jean-Pierre. C'est de là qu'il viendra, à la fin des années cinquante, pour préparer et obtenir à l'Université de Caen sa Licence ès Lettres et envisager ensuite des études de troisième cycle. Optant pour la géographie physique, plus particulièrement la géomorphologie, il bénéficiera des enseignements du professeur André Journaux et des maîtres-assistants de ce dernier Henri Elhaï et Yvette Dewolf. Ses premières recherches seront menées dans le cadre du Laboratoire de Géographie physique et du Centre de Géographie appliquée de l'Université de Caen créés en 1956 par A. Journaux. Il effectuera plusieurs missions en France, dirigées par Y. Dewolf, pour procéder à des relevés, à l'analyse et à la cartographie de formations superficielles, travaux préalables à des plans d'aménagement de communes rurales en déprise. Il profitera également des séminaires et stages de terrain de la RCP 77 consacrée à la cartographie géomorphologique et animée par $\mathrm{F}$. Joly.

Bien formé aux travaux de terrain, il entreprendra des recherches approfondies sur les formations superficielles du plateau du Caux qui aboutiront, en 1965, à la soutenance d'une thèse de troisième cycle intitulée "Les formations superficielles de SaintRomain-de-Colbosc (Pays de Caux). Leur contribution à l'étude géomorphologique de la région ». Remarquée par ses qualités, cette thèse sera publiée en 1968 dans la collection des Publications de la Faculté des Lettres et Sciences humaines de l'Université de Caen fondée par A. Journaux.

6 L'année 1965 marque aussi un tournant dans sa vie puisqu'il devient le premier attaché de recherche recruté par le tout nouveau Centre de Géomorphologie du CNRS, édifié 
sur le campus centre-ville de l'Université de Caen, dont les activités ont débuté pendant le dernier trimestre de 1964. Le doyen Journaux, directeur du Centre, lui confiera immédiatement l'animation et le développement du Laboratoire de cryoclastie prévu, dès l'origine, dans les plans de ce laboratoire de recherche.

7 C'est, sans nul doute, d'une part, de la présence sur son premier terrain d'étude de très belles coupes de lœss: Épouville, Goderville, Saint-Romain-de-Colbosc, liées à d'anciennes briqueteries et, d'autre part, des responsabilités qui viennent de lui être confiées au Centre de Géomorphologie que découleront les deux thèmes majeurs de ses recherches qui sont, pour l'un, l'étude des lœss de l'Europe du Nord-Ouest: faciès, paléopédologie, chronostratigraphie et, pour l'autre, la gélifraction des roches et l'étude des dépôts de pente périglaciaire. Cela ne l'empêchera pas de travailler sur d'autres sujets comme le système de nappes et de terrasses alluviales de la vallée de la Seine, les plages anciennes présentes sur le littoral normand et leurs couvertures de head et de lœss, certaines modalités de la remontée marine holocène et les dépôts tertiaires épandus sur les plateaux de la Haute-Normandie ou piégés dans les petits fossés tectoniques.

Dès l'achèvement de sa thèse de troisième cycle, Jean-Pierre Lautridou étendra sa zone d'étude des lœss à l'ensemble de la Haute-Normandie (Pays de Caux, basse vallée de la Seine) puis avec ses collaborateurs au Pays d'Auge, à la Campagne de Caen, enfin à la Normandie armoricaine. Il recueillera d'abondantes données lors des levés des 13 feuilles au 1/50 000 de la Carte géologique de la France auxquels il participera. Le second Bulletin du Centre de Géomorphologie sera, en 1968, consacré à son étude des lœess de Saint-Romain-de-Colbosc et de Mesnil-Esnard et, en 1969, dans un mémoire hors série de la Société géologique de France, il donnera une première synthèse sur les lœss du Pays de Caux. Il dressera une carte des épaisseurs des couvertures lœssiques, fortes en Haute-Normandie où les lœss anciens en particulier saaliens sont bien conservés et faibles dans la Normandie armoricaine, aux substrats imperméables et au relief très disséqué, où seuls les derniers dépôts (Weichselien) subsistent. Il cartographiera les variations de faciès des lœss et donnera une carte paléogéographique montrant les zones d'origine du lœss, ce matériau provenant essentiellement, selon ses études, du vannage des sédiments des paléoestuaires lors des fortes régressions marines accompagnant les maxima des froids quaternaires, les fonds de la Manche, mer épicontinentale étant alors totalement exondés. Découvrant pas moins de sept sols de rang interglaciaire, il se rendra compte que la pédostratigraphie des lœss sera un atout majeur pour construire un tableau chronostratigraphique du Pléistocène moyen et supérieur de la Normandie, tableau qu'il peaufinera sans cesse, la dernière version paraissant en 2009 dans le numéro de la revue Quaternaire dédié à la Normandie. Décrivant minutieusement les lœss weichseliens et leurs dépôts associés, traquant les moindres phases de pédogenèse ou d'érosion, celles-ci se traduisant par des horizons géliflués ou des cailloutis, il aboutira à une interprétation précise des fluctuations paléoenvironnementales au cours d'une période froide. D'où le titre de sa Thèse d'État, soutenue en 1984 et publiée en 1985 « Le cycle périglaciaire pléistocène en Europe du Nord-Ouest et plus particulièrement en Normandie ».

Jean-Pierre Lautridou sera amené à comparer puis à fusionner les résultats de ses travaux avec ceux entrepris en Belgique (R. Paepe), dans le Nord de la France (J. Sommé) et en Bretagne (J.-L. Monnier) dans le cadre du PICG « Quaternary glaciations in the Northern Hemisphere » et à participer aux travaux à la RCP « Lœss et formations 
associées " animée par J. Sommé et qui étendra son champ d'action à l'ensemble du Bassin de Paris et à l'Alsace (Achenheim). Sa réputation lui ouvrira les portes de Commission des lœss de l'INQUA dont il sera secrétaire de 1982 à 1986. Il continuera, jusqu'à la fin, à poursuivre ses investigations sur des coupes fondamentales comme celles de Saint-Pierre-lès-Elbeuf et d'Épouville profitant des nouvelles techniques qui apparaissaient et obtenant récemment pour Saint-Pierre des datations IRSL qui confirment ses propositions chronologiques antérieures basées sur la stratigraphie et la paleopédologie, la séquence couvrant 450000 ans.

D'aucuns ont voulu faire de Jean-Pierre un «Monsieur Lœss ». C'est bien vite oublier tout ce qu'il a entrepris dans le domaine de la cryoclastie expérimentale des roches et l'intérêt qu'il portait aux dynamiques périglaciaires actuelles des hautes latitudes et des hautes altitudes ainsi qu'aux dépôts et structures périglaciaires héritées des latitudes moyennes tels que les heads, les grèzes, les fentes de gel et coins de glace fossiles. Il était l'animateur de l'« Équipe Froid » du Centre de Géomorphologie.

Dans son laboratoire de cryoclastie, secondé par J.-C. Ozouf et Y. Delehaye, il a mené des expériences personnelles sur la microgélifraction des craies et de diverses roches du Massif armoricain, mais surtout, comme nous l'avons déjà dit, il a poursuivi de nombreuses expériences avec ses collègues de nombreuses universités françaises et avec des chercheurs étrangers. On peut dire que Jean-Pierre a brisé dans ses chambres froides toutes les roches de France et, en plus, bon nombre de roches venant du monde entier (Grande-Bretagne, pays scandinaves, Suisse, Afrique sub-saharienne et du Sud). Des échelles de gélivité ont été établies pour les roches calcaires et pour les roches siliceuses. C'est dans le domaine des roches calcaires que les études ont été poussées le plus loin. Avec G. Letavernier, étudiant en thèse, il a défini quatre classes de roches calcaires dont le type et la vitesse de fragmentation dépendent de leur porosité totale, de leur type de porosité, de leur perméabilité et de seuils dans la température de congélation de l'eau dans les pores. Ce sont: les roches microfissurées, les roches à pores moyens, les roches microporeuese, les roches perméables. En plus du bilan dressé dans sa thèse, il a publié deux articles importants, l'un dans la revue Progress in Physical Geography en 1982 « Experimental frost shattering: 15 years of research in the Centre de Géomorphologie du CNRS » l'autre "Recent advances in cryogenic weathering " paru en 1988 dans le volume "Advances in periglacial geomorphology " édité par M. J. Clark.

Sur le terrain, Jean-Pierre a suivi attentivement le programme Caen-Aix-en-Provence (P. Gabert) mené en Haute-Ubaye (Alpes du Sud) et axé sur la typologie et l'évolution actuelle des sols structuraux périglaciaires : sols striés, sols polygonaux ainsi que sur les vitesses des mouvements engendrés par la gélifluxion sur les versants entre 2700 et $3100 \mathrm{~m}$ d'altitude. Ce programme faisait suite à ceux de A. Cailleux et de A. Pissart avec lequel nous eûmes le plaisir de collaborer. Avec les membres de l'Equipe Froid, il étudiera les heads de Normandie dont la plupart sont weichseliens, mis en place entre 70000 et 55000 ans BP. Cependant, les grandes coupes de la Hague (Ecalgrain, Herquemoulin) révélèrent une double séquence avec un head et un lœss saaliens, un head et un lœss weichseliens. En conséquence, l'âge des plages anciennes de bas niveau situées en-dessous du être reconsidéré. Jean-Pierre Lautridou souhaita alors l'abandon du terme Normannien qui était utilisé pour désigner les plages du dernier interglaciaire situées légèrement au-dessus du niveau des plus hautes mers actuelles. Après de nouvelles études, la plupart des plages dont l'altitude est comprise entre $-1 \mathrm{~m}$ et $+7 \mathrm{~m}$ 
NGF purent être attribuées à l'Eemien (stade 5) mais plusieurs furent replacées dans une période régressive du stade 7 . Connaissant les travaux de Y. Guillien sur les grèzes des Charentes, il incitera J.-C. Ozouf à reprendre l'étude de ces dépôts en insistant sur la morphologie des gélifracts. La comparaison des gélifracts formant les grèzes avec ceux obtenus par le gel expérimental de blocs sains issus des calcaires de cette région montra que si l'expérience aboutit à une même homométrie et aux mêmes tailles des gélifracts, d'autres caractères ne sont pas respectés. Ces derniers prouve que le protocole expérimental tend à sous estimer le rôle de la microfissuration des roches par la tectonique et celui de l'altération chimique en période tempérée.

La reconnaissance de ses travaux conduira Jean-Pierre Lautridou à la présidence de la Commission du périglaciaire de l'Union géographique internationale (1988-1996) et à celle du Groupe de travail périglaciaire de l'Association internationale du pergélisol (1988-1993).

En 1979, Jean-Pierre Lautridou organise le Groupe Seine, multidisciplinaire, comprenant des géomorphologues, des géologues, des paléontologues, des palynologues, des malacologues et des préhistoriens. Six bulletins du Centre de Géomorphologie, dont il put assurer la parution jusqu'en 1993, relateront les travaux du groupe qui concernent le système des nappes et terrasses fluviatile de la Seine, la paléontologie des formations quaternaires dans le domaine Normandie-Manche (colloque de Rouen 1986), les tufs et travertins des bassins de la Seins et de la Somme et des régions limitrophes (colloque de Rouen 1984), le Quaternaire et la préhistoire des métropoles normandes.

Depuis son premier enfoncement dans les plateaux, la Seine a laissé 10 ensembles étagés de nappes et de terrasses couvrant environ un million d'années. Une belle synthèse collective a été publiée en 1999 dans le Bulletin de la Société géologique de France sous le titre «Le fleuve Seine. Le fleuve Manche ». Deux sites ont été au centre des préoccupations du groupe: Tourville-la-Rivière et Tancarville en raison de la présence de paléoestuaires. Tancarville possède aussi une paléodune. Les études paléontologiques et des datations ESR et IRSL ont permis d'attribuer les paléoestuaires de Tourville-la-Rivière aux stades 7 et 9 . La paléodune de Tancarville date de l'Eemien et le paléoestuaire du stade 7 . Une synthèse des connaissances peut être trouvée dans l'article de P. Antoine, J.-P. Lautridou et M. Laurent paru dans Geomorphology en 2000 "Long-term fluvial archives in NW France, response of Seine and Somme rivers to tectonic movements, climatic variations and sea-level changes ».

16 Petit à petit, les progrès dans les connaissances sur le Quaternaire de Normandie ont permis un calage des formations par rapport aux biozones définies internationalement et par rapport aux données issues des carottes océaniques ou glaciaires.

17 Après avoir publié quelques articles sur les argiles à silex de Haute-Normandie, JeanPierre reprendra avec divers collègues l'étude des dépôts de la fin du tertiaire et du Pléistocène inférieur avec en Haute-Normandie les sites de La Londe, Saint-Eustache, Valmont et des formations comme les Sables de Lozère et en Basse-Normandie les formations su Seuil du Cotentin. Citons l'article paru en 1979 dans le Bulletin des géologues du Bassin de Paris « G. Kuntz et J.-P. Lautridou. Le Plio-Pléistocène de HauteNormandie » et le bilan dressé avec O. Dugué et M. Clet dans le numéro de la revue Quaternaire consacré à la Normandie en 2009 sous le titre "Évolution sédimentaire cénozoïque (Paléocène à Pléistocène inférieur) de la Normandie ». 
Le Groupe Manche était en peine activité lorsque Jean-Pierre s'est vu dans l'obligation de mettre fin progressivement à son activité scientifique. La première phase des recherches qui consistait à faire la mise au point la plus exhaustive possible des connaissances sur la mer de la Manche et les régions adjacentes était achevée. Quatorze articles sont parus dans la revue Journal of Quaternary Science. Ils portaient sur des sujets très variés : tectonique, vallées continentales et sous-marines, canyons et deltas profonds, fosses, formations quaternaires des reliefs (argiles à silex, lœss, dépôts de pente), plages perchées et paléoestuaires pléistocènes, pergélisol fossile, remontée marine holocène. Jean-Pierre était co-auteur de cinq de ces articles.

Compte tenu de ses connaissances encyclopédiques sur le Quaternaire de la Normandie, il était inévitable qu'il poursuive une collaboration permanente et intense avec les préhistoriens et protohistoriens de cette province sans négliger pour autant des incursions dans d'autres régions comme la Bretagne, lî̂le-de-France et la Picardie.

Cette collaboration débute en 1968 après l'acquisition par l'État de la plus grande carrière de Saint-Pierre-lès-Elbeuf et surtout la reprise des investigations avec G. Verron, Directeur des Antiquités préhistoriques de Normandie $(1970,1974)$. Puis se mettent en place des partenariats dans un premier temps ponctuels avec la conduite d'études géomorphologiques et environnementales menées à partir de la fin des années 1960 sur les sites paléontologiques et préhistoriques de Tourville-la-Rivière et de Cléon (Seine-Maritime) avec G. Carpentier, puis le début des années 1970, avec l'étude des sites de Goderville (Seine-Maritime) de 1972 à 1976, avec G. Fosse et G. Verron, d'Acquigny avec G Fosse, de Tancarville (Seine-Maritime) en 1983 avec B. Masson et P. Lebret, etc.

21 Cette pluridisciplinarité se structure avec la constitution du Groupe Seine, dont le domaine d'intervention de la vallée de Seine (1979-1993) et des plateaux qui l'encadrent déborde largement la Normandie (travaux engagés avec Y. Dewolf, F. Lécolle), puis du Groupe l'Homme et la mer, animé par G. Fosse (1984-1986), qui analyse les interactions entre l'Homme paléolithique et son milieu en contexte littoral. Ce programme de recherche se développe autour de travaux de fouilles de sauvetages et de fouilles programmées conduites dans le nord du département de la Manche, par D. Michel à Montfarville/La Houe, à Saint-Vaast-la-Hougue/Le Crau à Fermanville/Port-Pignot, à Saint-Germain-des-Vaux/Gélétan, par G. Vilgrain à Gouberville, par G. Fosse à SaintVaast-la-Hougue/Le Fort et par D. Cliquet à Saint-Germain-des-Vaux/Port-Racine. L'analyse de ce dernier site participera largement aux travaux portant sur les formations littorales développés dans le cadre de sa Thèse d'État soutenue en 1984.

2 Enfin, c'est avec la constitution du projet collectif de recherche « les premiers Hommes en Normandie » animé par D. Cliquet (depuis 2000) que l'interdisciplinarité prend toute sa dimension, avec la reprise de sites anciennement fouillées, la constitution d'un cadre chronologique fondé sur les méthodes radiométriques dont les premiers résultats paraissent dans deux numéros de Quaternaire consacrés à la Normandie (2003 et 2009).

C'est dans le cadre de ce projet collectif que Jean-Pierre partage sa passion avec nombre de bénévoles, initie profanes et étudiants à la géomorphologie, s'implique dans l'étude des contextes environnementaux pléistocènes des deux Normandie. De 2000 jusqu'à ce que la maladie le prive du terrain en 2008, Jean-Pierre participe à toutes les opérations archéologiques conduites sur les occupations paléolithiques de Normandie. C'est pour lui l'occasion de reprendre l'analyse des sites de Saint-Pierre-lès-Elbeuf, de Tancarville, de Tourville, en Seine-Maritime, d'Écalgrain, dans la Manche, de Glos, dans le Calvados, 
de s'ouvrir à de nouveaux horizons avec les fouilles des sites moustériens de Rânes dans l'Orne et du gisement belloisien de Rouvres dans le Calvados.

Cependant son implication dans la recherche archéologique ne se limite pas au Pléistocène, comme en témoignent les études menées sur les sites holocènes de la frange littorale des côtes de la Manche. Il participe au projet collectif de recherche « l'exploitation des milieux littoraux en Basse-Normandie » dirigé par C. Billard avec le suivi des pêcheries protohistorique de Saint-Jean-le-Thomas et médiévale de Saint-Lôd'Ourville (Manche).

Parallèlement il intervient ponctuellement sur les sites holocènes de Lingreville (Manche) et de Biéville-Beuville (Calvados), avec C. Billard, de Graye-sur-Mer et Asnelles (Calvados) avec J. Couvelard.

L'œuvre de Jean-Pierre Lautridou est considérable. En plus de ses thèses de Troisième cycle et d'État, il a publié environ 220 articles dont le quart reconnu de rang A, articles de référence fréquemment cités par les chercheurs du monde entier. Beaucoup ont été publiés dans des revues importantes telles que Quaternaire, Quaternary Sceince Revews, Journal of Quaternary Science, Permafrost and Periglacial Processes. Il a été lui-même membre de nombreux comités éditoriaux. Il a beaucoup fait pour le rayonnement national et international du Centre de Géomorphologie du CNRS où il $\mathrm{a}$ mené toute sa carrière et dont il a été directeur de 1984 à 1992. Par ses fonctions au niveau international, il a contribué, plus largement, à la reconnaissance des géosciences françaises à l'étranger. Tu nous as fait bénéficier, tout au long de nos propres carrières de ta gentillesse et de tes grandes connaissances scientifiques. Merci Jean-Pierre. Ton départ laisse un grand vide dans la communauté scientifique à laquelle tu appartenais, tristesse et désarroi dans le cœur de ceux qui t'ont connu et travaillé avec toi.

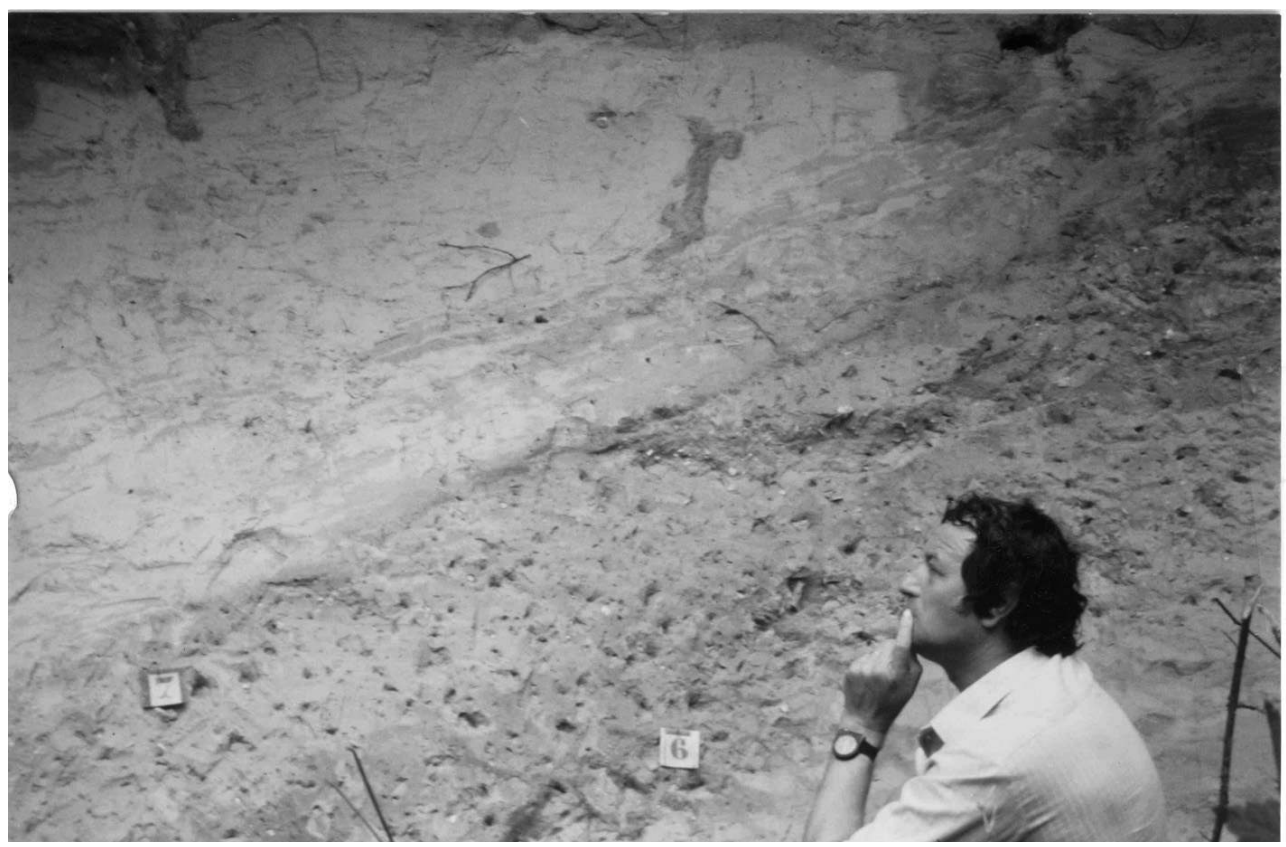

Examen de la dune éémienne de Tancarville 


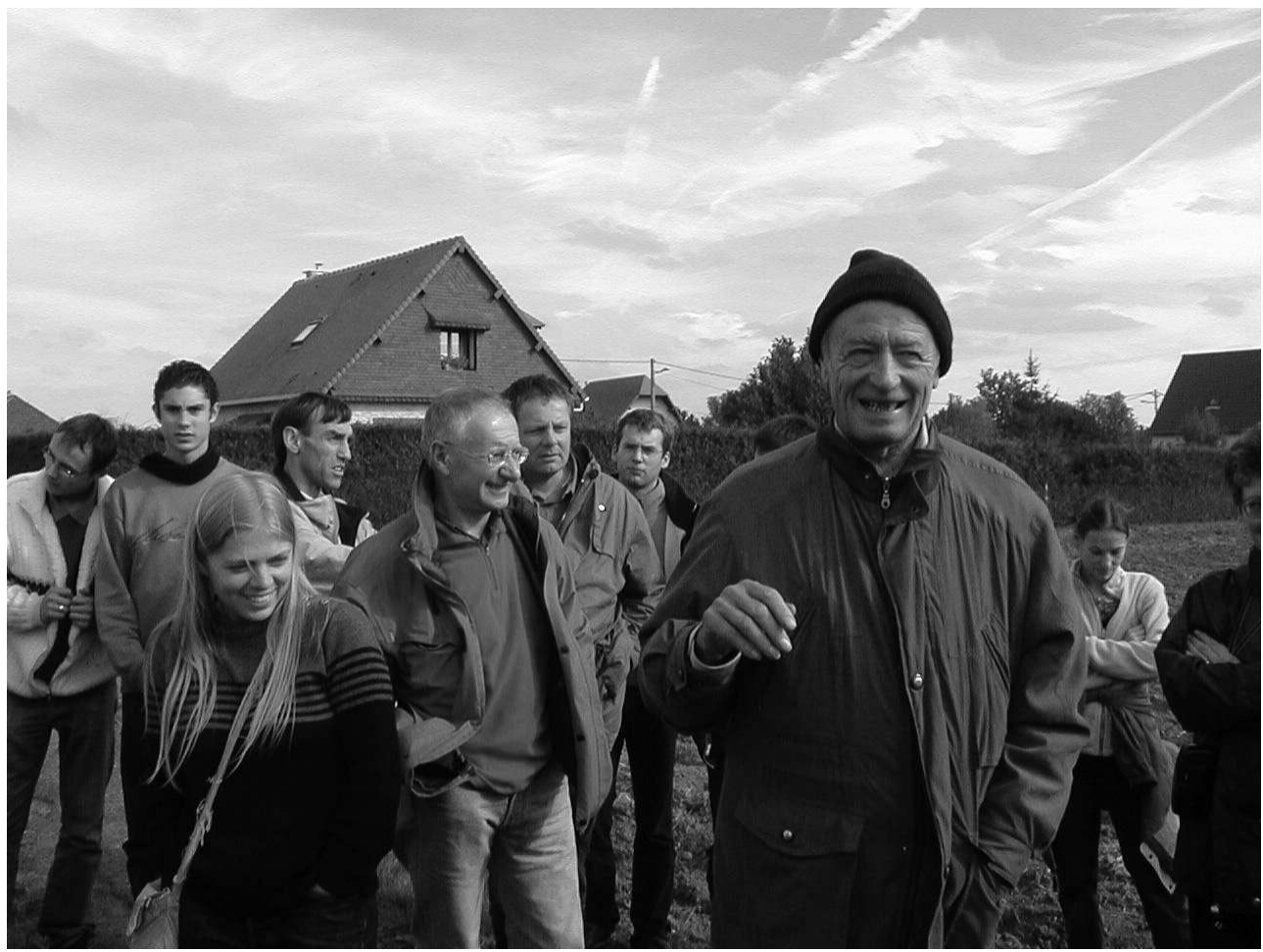

Visite de site avec l'association PAN en octobre 2003 